\title{
Importance of matrix effects in LC-MS/MS bioanalysis
}

\author{
"According to our experience, the matrix effect should always be carefully and thoroughly examined as it may be \\ revealed in diverse and unexpected forms impacting accuracy of the data generated."
}

Keywords: hemolyzed plasma $\approx$ LC-MS/MS $\approx$ matrix effects $\approx$ phospholipids $\approx$ stability

The matrix effect has been discussed in many publications over the years since its first report in a bioanalytical assay by Buhrman et al. in 1996 [1]. This phenomenon established by Kebarle et al. in 1993 is generally defined in LC-MS/MS as the impact of the co-eluting compounds endogenously present in the matrix on the ionization efficiency and the reproducibility of the ionization source [2]. Due to the high importance of the matrix effect on the reliability of data generated in LC-MS/MS bioanalysis, the regulatory agencies require demonstration of no impact coming from the matrix as part of a bioanalytical method validation.

A review of the literature on matrix effect brings up discussion on different aspects of this phenomenon [3-5]. A choice of procedures are suggested to assess the matrix effect; postextraction spike method, postcolumn infusion method, phospholipids monitoring, and so on. Numerous causes of the matrix effect, such as phospholipids, and several specific cases of matrix effect observed in bioanalytical methods are investigated. Moreover, various well known solutions to circumvent the matrix effect are published; stable labeled IS, cleaner sample preparation, chromatographic separation from the interference matrix component, and so on. It is also possible to find innovative techniques and instrumentation that boast themselves about being able to reduce or even eliminate the matrix effect in the LC-MS/MS bioanalysis field, such as phospholipids plate removal and ion mobility instrument.

This Special Focus Issue on matrix effect reports multiple real-life cases encountered in the bioanalytical industry, several that are frequently observed and others as infrequent as the erroneous stability assessment. In parallel, various solutions found by the authors to circumvent this phenomenon are described; ion mobility utilization, ionization polarity and mode change, stabilization agents, and so on. Some method practical tips for development are proposed to proactively assess the matrix effect before bioanalysis such as the use of fresh matrix or the dosing vehicle and stabilization agent impact assessment. In addition to the ionization/enhancement common category of matrix effect, also discussed are the isobaric interference and the matrix components that modify the extraction recovery of the analyte by protein binding or other. The pros and cons in terms of matrix effect of a stable labeled IS, reverse-phase and HILIC chromatography along with the type of sample preparation (protein precipitation, liquid-liquid and SPE) are also evaluated, including the potential of SPE for the removal of background peptides during therapeutic protein analysis via pellet tryptic digestion assays. In addition, this issue also includes an interesting discussion on tissue analysis particularity in terms of assessing and solving matrix effect, due to the complex properties of the tissue itself. Moreover, whereas matrix commonly mean sample matrix (i.e., plasma, blood, and so on) in the industry, the mobile phase is the major component of the matrix entering the mass spectrometer with the analyte. Regarding this topic, an uncommon investigation of the mobile phase impact on ionization effect and retention time of LC-MS/MS methods is reported. Therefore, due to its varied content, this Special Focus Issue is believed to be of real and high interest for one who works on LC-MS/MS bioanalysis in a controlled regulated environment.

In bioanalysis, the matrix effect is an important occurrence, even in the presence of stable labeled IS that cannot be forgotten when it comes to method development. According to our experience, the matrix effect should always be carefully and thoroughly examined as it may be revealed in diverse and unexpected forms

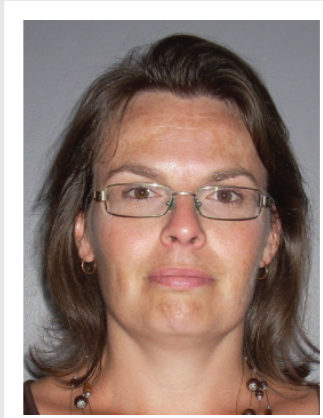

Annik Bergeron

Algorithme Pharma Inc. 575, boul. Armand-Frappier, Laval, Québec, H7V 4B3, Canada

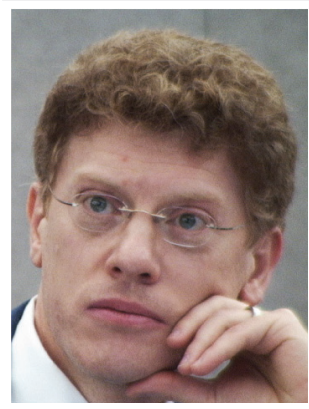

Fabio Garofolo

Author for correspondence: Algorithme Pharma Inc. 575, boul. Armand-Frappier, Laval, Québec, H7V 4B3, Canada

Tel.: + | 4509736077

E-mail: fgarofolo@algopharm.com 
impacting accuracy of the data generated. See for instance the following three recent investigations: matrix effect in HILIC LC-MS/MS assays due to phospholipids [6]; hemolyzed plasma endogenous components leading to instability $[7,8]$ and long-term stability issue due to presence of organic solvent in matrix [9].

Since HILIC chromatography is now widely used in a bioanalytical environment, this matrix effect investigation is of interest [6]. Indeed, this case was performed in order to determine the effect of HILIC chromatography and its particular operating conditions on the retention of the phospholipids, reported as the main cause of matrix effect. A thorough evaluation of the phospholipids using multiple HILIC columns has demonstrated that lysophosphatidylcholine and phosphatidylcholine phospholipids represent a major source of ionization effect in HILIC chromatography. Among the tested HILIC columns, the silica- and diol-based columns were found to have higher possibility of late ionization effect due to the lysophosphatidylcholine phospholipids late elution.

The second investigation was executed when stability issue of phenolic compounds in hemolyzed plasma was observed $[7,8]$. During morphine method development, processed reconstituted instability was demonstrated in hemolyzed plasma whereas in 'normal' plasma the analyte was stable. Following laborious and exhaustive testing, the degradation was found to result from radical oxidation of the ionized phenolic group promoted by the presence of methemoglobin found in hemolyzed plasma. This problem would not have been identified if the hemolyzed plasma was not part of matrix effect evaluation regularly performed during method developments.
Finally, published as part of this Special Focus Issue, a case observed in our laboratory where matrix effect was encountered in samples fortified with co-administered drug (CAD) [9]. As required by regulatory agencies to demonstrate the selectivity of the bioanalytical method, samples were fortified with both the analyte and the CAD. Long-term instability of the CAD fortified samples was observed, whereas other samples met the acceptance criteria. After multiple experiments, it was found that the matrix effect causing this instability was due to the small amount of organic solvent added during the fortification with the CAD which creates suppression over time.

Up until now, no one has found a universal solution to avoid matrix effect in bioanalysis since it may be present in too many different forms and it is frequently drug-dependent. Therefore, bioanalysts will have to continue improving their way to investigate matrix effect and searching for innovative solutions to circumvent this important and not always easily observable issue. For this reason, we would like to see the publication of a new Special Focus Issue dedicated to matrix effect in LC-MS/MS in the near future from the present one, fuelled by novel state-of-the-art approaches and new interesting case studies on this never-ending issue in bioanalysis.

\section{Financial \& competing interests disclosure}

The authors have no relevant affiliations or financial involvement with any organization or entity with a financial interest in or financial conflict with the subject matter or materials discussed in the manuscript. This includes employment, consultancies, honoraria, stock ownership or options, expert testimony, grants or patents received or pending, or royalties. No writing assistance was utilized in the production of this manuscript.

\section{References}

1 Buhrman DL, Price PI, Rudewicz PJ. Quantification of SR 27417 in human plasma using electrospray liquid chromatographytandem mass spectrometry: a study of ion suppression. J. Am. Soc. Mass. Spectrom. 7, 1099-1105 (1996).

2 Kebarle P, Tang L. From ions in solution to ions in the gas phase - the mechanism of electrospray mass spectrometry. Anal. Chem. 65(22), 972A-986A (1993).

3 Taylor PJ. Matrix effects: the Achilles heel of quantitative high-performance liquid chromatography-electrospray-tandem mass spectrometry. Clin. Biochem. 38(4), 328-334 (2005).
4 Van Eeckhaut A, Lanckmans K, Sarre S, Smolders I, Michotte Y. Validation of bioanalytical LC-MS/MS assays: evaluation of matrix effects. J. Chromatogr. B Analyt. Technol. Biomed. Life Sci. 877(23), 2198-2207 (2009).

5 Côté C, Bergeron A, Mess J-N, Furtado M, Garofolo F. Matrix effect elimination during LC-MS/MS bioanalytical method development: a review. Bioanalysis 1(7), 1243-1257 (2009).

6 Mess J-N, Côté C, Bergeron A, Furado M, Garofolo F. Selection of HILIC columns to handle matrix effect due to phospholipids. Bioanalysis 1(1), 57-62 (2009).
7 Bérubé E-R, Taillon M-P, Furtado M, Garofolo F. Impact of sample hemolysis on drug stability in regulated bioanalysis. Bioanalysis 3(18), 2097-2105 (2011).

8 Bérubé E-R, Lacasse M-C, Furtado M, Garofolo F. Severe impact of hemolysis on stability of phenolic compounds. Bioanalysis 5(12), 1491-1499 (2013).

9 Dicaire C, Mess J-N, Furtado M, Bergeron A, Garofolo F. Impact of organic solvent additive on the integrity of plasma samples in bioanalysis by LC-MS/MS. Bioanalysis 5(19), 2359-2370 (2013). 